\title{
Optically Enhanced Photon Recycling in Mechanically Stacked Multijunction Solar Cells
}

\author{
Myles A. Steiner, John F. Geisz, J. Scott Ward, Iván García, Daniel J. Friedman, Richard R. King, Philip T. Chiu, \\ Ryan M. France, Anna Duda, Waldo J. Olavarria, Michelle Young, and Sarah R. Kurtz
}

\begin{abstract}
Multijunction solar cells can be fabricated by mechanically bonding together component cells that are grown separately. Here, we present four-junction four-terminal mechanical stacks composed of GaInP/GaAs tandems grown on GaAs substrates and GaInAsP/GaInAs tandems grown on InP substrates. The component cells were bonded together with a low-index transparent epoxy that acts as an angularly selective reflector to the GaAs bandedge luminescence, while simultaneously transmitting nearly all of the subbandgap light. As determined by electroluminescence measurements and optical modeling, the GaAs subcell demonstrates a higher internal radiative limit and, thus, higher subcell voltage, compared with GaAs subcells without the epoxy reflector. The best cells demonstrate $38.8 \pm 1.0 \%$ efficiency under the global spectrum at $1000 \mathrm{~W} / \mathrm{m}^{2}$ and $\sim 42 \%$ under the direct spectrum at $\sim 100$ suns. Eliminating the series resistance is the key challenge for further improving the concentrator cells.
\end{abstract}

Index Terms-Luminescent coupling, mechanical stack, multijunction solar cell, photon recycling, III-V solar cell.

\section{INTRODUCTION}

$\mathbf{T}$ HE detailed balance limit for the conversion efficiency of a solar cell implicitly assumes the maximum degree of photon recycling: With the device biased at open circuit, every electron-hole pair recombines radiatively to produce a photon near the bandedge, and all photons that are not emitted out the front of the cell are reabsorbed to generate a new electron-hole pair. The steady-state density of photons in the absorber increases, which correspondingly increases the density of minority carriers and therefore $V_{o c}$. Relative to a case where photon recycling is fully suppressed, $V_{o c}$ for GaAs-like cells can increase by up to $70 \mathrm{mV}$ at room temperature.

Real solar cells fail to reach the detailed balance limit for several reasons. Neglecting losses due to series and shunt resistances, the main losses result from the fact that 1) the recombination of all electron-hole pairs is never completely radiative, and 2) some of the internally emitted photons are reabsorbed parasitically and lost. The first loss can be addressed primarily by minimizing the nonradiative Shockley-Read-Hall recombination rates in the quasi-neutral and depletion regions, which are determined by the density of defects such as the background concentration of oxygen, as well as the band structure of the device. Auger recombination can be significant in $\mathrm{Si}$ cells but only begins to dominate III-V cells at very high injection levels.

The second loss, due to parasitic absorption of emitted photons, is more difficult to address and has been the subject of several recent reports [1]-[3]. Photons are emitted isotropically within the absorber layer and eventually encounter interfaces between the semiconductor layers, where the indexes of refraction are never exactly matched, and the resulting reflection or transmission coefficients are always less than unity. In some cases, the various layers surrounding the absorber may also have nonzero extinction coefficients such that they are not strictly transparent. A prime example is the GaAs or Ge substrate adjacent to a III-V solar cell, which acts as a nonradiative recombination sink to most of the photons that are transmitted into it.

Recent work on GaAs, GaInP, and GaInAs solar cells has demonstrated that by removing the GaAs or InP substrate and depositing a highly reflective mirror, a large fraction of the photons that would have been absorbed in the substrate are reflected back toward the absorber where they are "recycled." The effect can be observed by measuring both a higher $V_{\mathrm{OC}}$ and a higher external radiative efficiency (ERE) and has lead to record efficiencies in GaAs [4] and GaInP [5] solar cells. Those cells used metallic mirrors to reflect the luminescence, but a low-index transparent material such as epoxy would also be suitable. Fig. 1 shows the modeled angle- and wavelengthdependent reflectance at the back interface of a GaInP/GaAs tandem cell, for cells backed by GaAs or by epoxy. With the GaInP back-surface field layer and the $\mathrm{AlGaAs}$ contact layer on a GaAs substrate, the escape cone is very wide, i.e., $\sim 67^{\circ}$, and the reflectance is $<10 \%$ at most angles; the angle- and energyaverage reflectance is $\sim 21 \%$. With the epoxy reflector the escape cone narrows to $\sim 24^{\circ}$ and the average reflectance increases to $\sim 83 \%$ because any luminescence that strikes the interface outside the escape cone is totally internally reflected. An air interface would also be suitable, providing an even narrower escape cone than the epoxy [6]. Based on the model described 


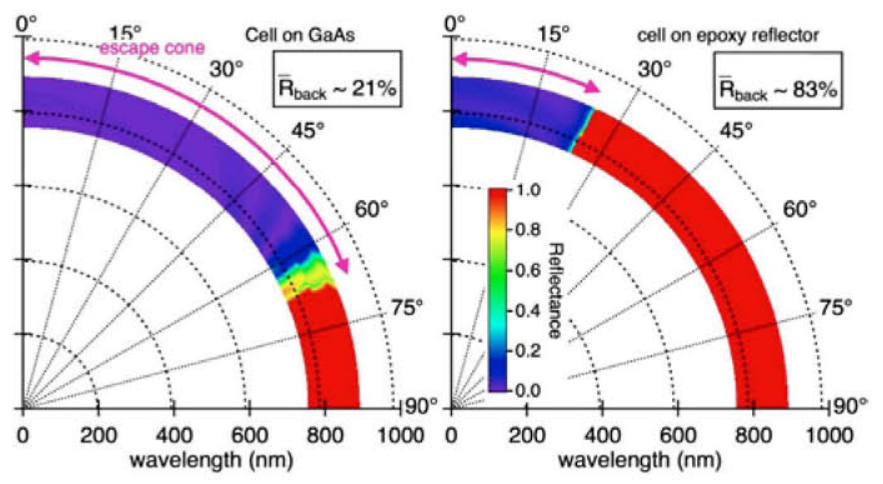

Fig. 1. Modeled reflectance at the interface behind the GaAs junction of a GaInP/GaAs tandem for devices backed by a GaAs substrate (left) and a $\mathrm{ZnS} /$ epoxy handle (right). Only the wavelengths near the GaAs emission are shown. The pink arcs indicate the escape cone, where most of the luminescence is not reflected. The angle- and energy-averaged reflectances at the back of the cell are shown in the boxes.

in [1], $V_{\text {oc }}$ should improve from $1.063 \mathrm{~V}$ with the GaAs substrate to $1.096 \mathrm{~V}$ with the epoxy reflector, and up to $1.108 \mathrm{~V}$ with a gold reflector, all assuming perfect internal radiative efficiency (IRE).

In principle, comparable enhancement of photon recycling is possible in multijunction solar cells by the insertion of selective photon reflectors at the back of each junction [7] but is more challenging than in single-junction cells because of the inaccessibility of the rear surface of all but the bottom junction. Further complicating the design, the selective photon reflector has to reflect a very high fraction of the luminescent photons in a particular junction but transmit all of the subbandgap photons as well so that the adjacent lower bandgap junctions are not starved of light. Previously considered strategies such as the inclusion of a monolithically grown distributed Bragg reflector [8] or laterally oxidizing a buried AlAs layer to form an aluminum oxide layer with a refractive index of $\sim 1.7$ [9], both suffered from the inability to simultaneously reflect the luminescent light and transmit nearly all of the subbandgap light.

Forming a multijunction solar cell by mechanically stacking the component junctions presents a promising pathway to photon recycling enhancement, because the accessibility of the intermediate interfaces naturally invites the inclusion of selective optical reflectors. A low-index layer such as transparent flip-chip epoxy $(n \sim 1.5)$ acts as a reflector [6] to the luminescence as shown in Fig. 1, and additional $\mathrm{ZnS}$ intermediateindex dielectric films on either side of the epoxy maximize the transmission of normal-incidence subbandgap light. Mechanical stacks have several other attractive features and have recently been demonstrated in different forms by several groups [10][13]. Because the subcells are grown separately, the problems associated with lattice-mismatch [14] and thermal-expansion mismatch are nearly eliminated. Indeed, one could form a mechanical stack from dissimilar material systems (e.g., III-V and II-VI alloys, or silicon [15]) in a way that would be challenging on a monolithic epitaxial platform. These benefits of a mechanical stack with photon recycling-enhancing optical interlayers must be weighed against the disadvantages of additional cost and complexity associated with the processing of such a structure. They must also be weighted against the benefits of luminescent coupling, where excess photocurrent in an upper junction is recovered in a lower junction through the emission and reabsorption of photons, which helps mitigate spectral sensitivity [16]-[18].

In this paper, we demonstrate enhanced photon recycling in mechanical stacks by using a low-index transparent epoxy to bond the subcells. Based on component cells that were grown separately on GaAs and InP substrates, we have fabricated devices that are electrically interconnected in four-terminal configurations, thereby eliminating the need for a conductive epoxy. The four-terminal configuration has the additional advantages of lessening the constraints on current matching and potentially increasing the annual energy yield. We compare the electrical and optical characteristics to those of a two-terminal four-junction inverted metamorphic multijunction (IMM) cell [14].

\section{Solar Cell Fabrication}

The four-junction devices described here consist of an upper $\mathrm{GaInP} / \mathrm{GaAs}(1.85 / 1.42 \mathrm{eV})$ tandem grown at NREL and a lower GaInAsP/GaInAs (1.05/0.74 eV) tandem grown at Spectrolab. The upper tandem was grown inverted and the substrate was removed as part of the processing [19]. The lower tandem was grown upright on n-type InP and the substrate remained part of the final structure; this tandem is a version of the one included in the 5J wafer-bonded cell described in [12]. The process can be adapted to include an inverted bottom tandem.

To process the device, first the bottom tandem was grown and fully processed, including metallization and mesa isolation. Grids were then deposited on the back of the upper tandem, $\mathrm{ZnS}$ antireflection coatings (with thicknesses as modeled and discussed below) were deposited by thermal evaporation on the back of the upper tandem and the front of the lower tandem, and the two components were bonded together with $\sim 10 \mu \mathrm{m}$ of epoxy; the epoxy layer is optically thick and the precise thickness is not important. The GaAs substrate was then removed from the upper tandem by wet chemical etching. Finally, the front grids were electroplated, the upper tandem was mesaisolated, and the four-layer front antireflection coating was thermally deposited.

Fig. 2 shows a schematic and a composite optical image of the concentrator version of the mechanical stack. The upper mesa and front grids are clearly visible in the photograph, but other buried features can also be discerned. The structure was designed so that the lower tandem would have a mesa area roughly twice that of the upper tandem, to accommodate any misalignments during the bonding process. The upper tandem mesa and gridded back contact act as an aperture to the lower tandem so that all four junctions have the same illuminated area. The wide busbars on the front of the third junction, which are visible in the photograph in Fig. 2, are therefore positioned outside the aperture area so that they do not contribute additional shadowing. A long tab extends off the side of the lower tandem, as shown in Fig. 2, allowing the third contact metal to protrude from beneath the upper tandem for external access. The grids of the first and second contacts are aligned, but the third 

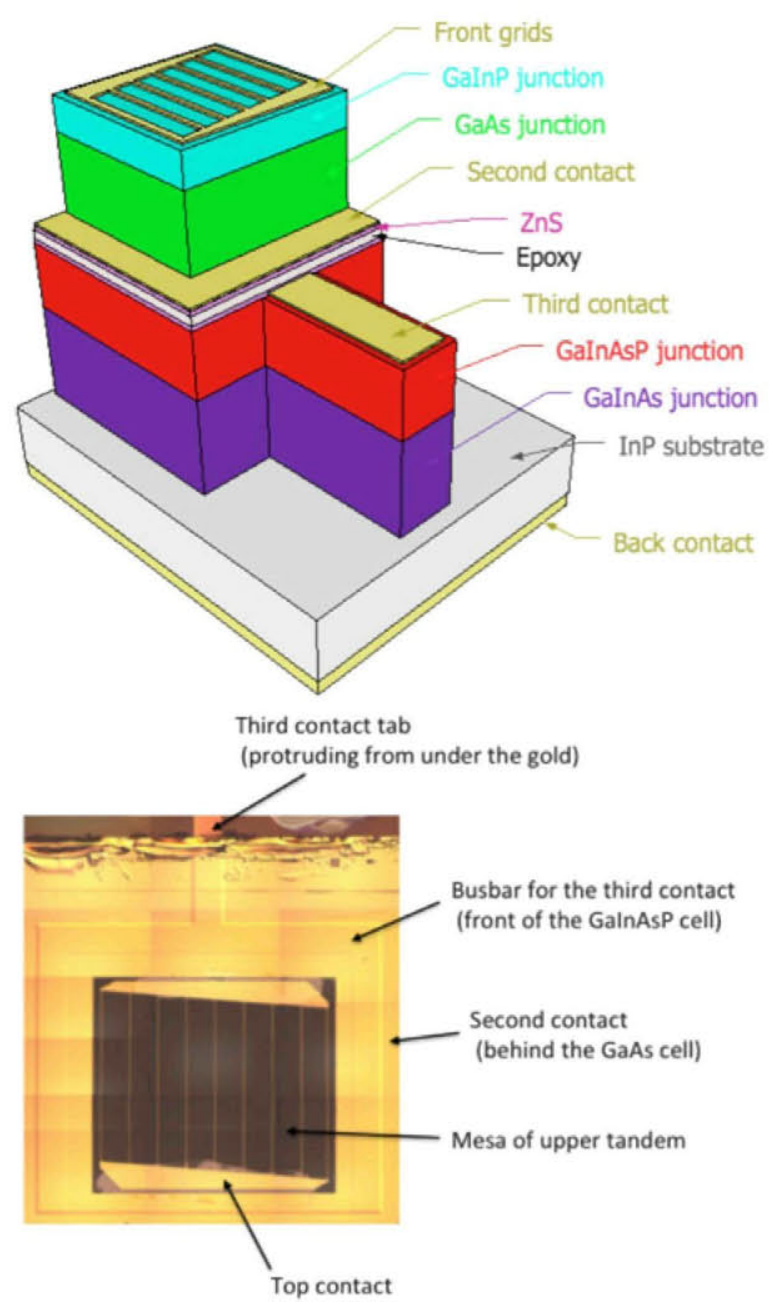

Fig. 2. (Top) Three-dimensional schematic of the four-junction mechanical stack layer structure (not to scale) on an InP substrate. For clarity, some layers are not shown. (Bottom) Nomarski composite image of the mechanically stacked solar cell. Buried features can be seen because the variation in thickness creates visual contrast. The mesa of the upper tandem is aligned with the aperture behind the second junction and fits well within the busbar of the third junction. External contact to the third junction is made on the protruding tab shown at the top of the image. The fourth contact is made to the back of the n-type InP wafer.

contact grids are not aligned to the upper grids, and therefore, the shadowing is slightly increased; flip-chip processing equipment could enable improved alignment between the upper and lower grids. The fourth contact is made to the back of the n-type InP substrate. Thick transparent lateral conduction layers were grown behind the GaAs cell and in front of the GaInAsP cell. The two tandems are separated by a low-viscosity transparent epoxy with a refractive index of $\sim 1.5$ (light pink layer in Fig. 2), and on either side of the epoxy, we deposited ZnS (dark pink layers) to increase the transmission of incident light to the lower tandem. Sandwiched between semiconductor layers with $n \sim$ 3.6 and an epoxy with $n \sim 1.5$, the ideal single layer intermediate ARC would have an index of $\sqrt{3.6 \times 1.5}=2.3$, and $\mathrm{ZnS}$ has an index of $\sim 2.4$, which is close to ideal.

Since the ZnS layers act as an intermediate antireflection coating, the thickness can have a large effect on the device performance. Fig. 3 shows the modeled effect of the $\mathrm{ZnS}$ thick-

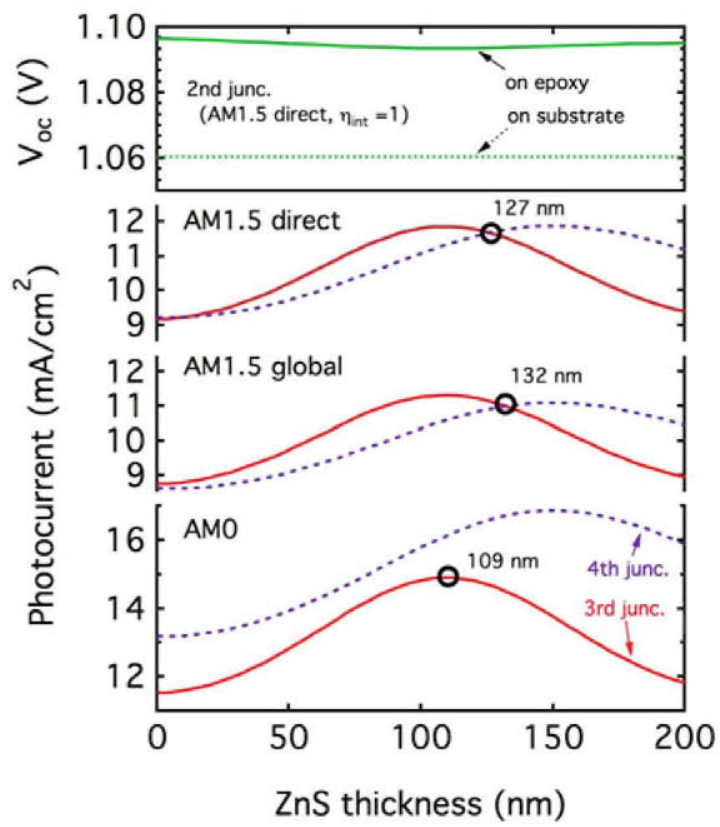

Fig. 3. Modeled effect of the $\mathrm{ZnS}$ intermediate antireflection coating on the $V_{o c}$ of the GaAs junction under the direct spectrum (upper panel), and the photocurrents of the GaInAsP and GaInAs junctions under three reference spectra (lower panel). Solid red lines shows the GaInAsP third junction; dashed purple lines show the GaInAs fourth junction. The black circles indicate the optimum thickness in each case. The model is based on a transfer matrix calculation of electromagnetic waves through the upper junctions and assumes a four-layer antireflection coating on the front surface and appropriate thicknesses for the semiconductor layers in the upper junctions. For the $V_{\text {oc }}$ model, we assume an IRE of unity, for simplicity. The dotted line in the upper panel shows the modeled $V_{o c}$ of the GaAs junction if it were still on the substrate, with no epoxy reflector or $\mathrm{ZnS}$ intermediate $\mathrm{ARC}$.

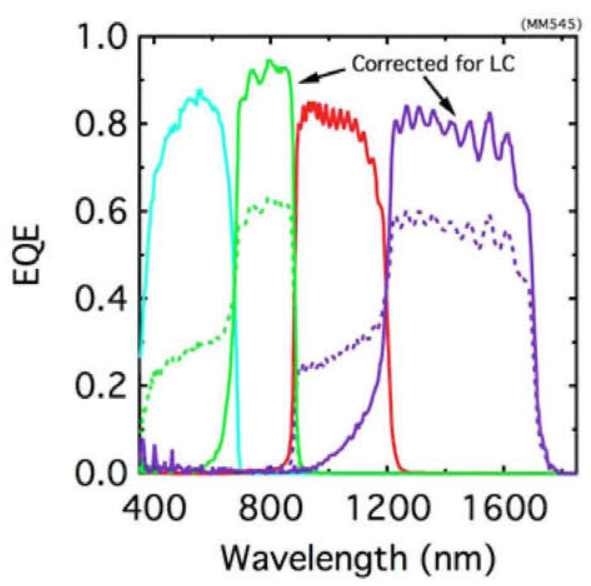

Fig. 4. EQE for a 4J 1-sun mechanical stack. The dashed lines show the raw data for the second and fourth junctions, showing strong luminescent coupling from the first and third junctions, respectively. The data were corrected following the method in [26].

ness on the photocurrents of the bottom junctions under three spectra, as well as $V_{\text {oc }}$ of the GaAs junction under the direct spectrum. The model is based on the measured external quantum efficiency (EQE) curves for a GaInAsP/GaInAs tandem as shown in Fig. 4 and the calculated transmission of incident light through a GaInP/GaAs/ZnS/epoxy/ZnS structure. Based on the specific structures considered here, the ZnS thickness can affect 


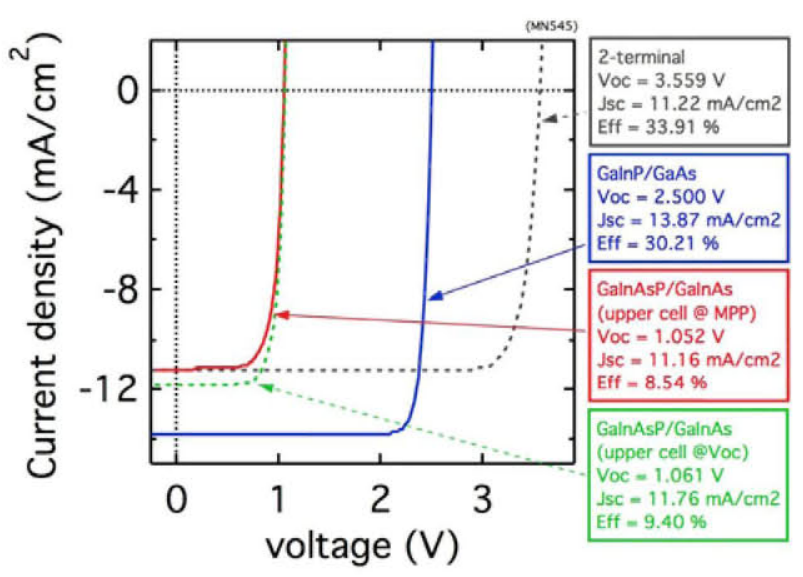

Fig. 5. NREL-certified $I-V$ curves acquired on an adjustable solar simulator, under a spectrum equivalent to AM $1.5 \mathrm{G} 173$ global at $1000 \mathrm{~W} / \mathrm{m}^{2}$. The blue curve shows the upper tandem. The red curve shows the lower tandem with the upper tandem biased at $V_{\mathrm{mpp}}$. The green-dashed curve shows the lower tandem with the upper tandem biased at $V_{o c}$. The black-dashed curve shows two-terminal $I-V$ characteristic, measured by shorting the second and third terminals.

which junction is limiting under the global and direct spectra. Neglecting any additional photocurrent generated by luminescent coupling from the GaAs junction, the optimum thickness is clearly at the crossover point, indicated by the circles in the figure. For the AM0 spectrum, the third (GaInAsP) junction appears to always be limiting, and the $\mathrm{ZnS}$ thickness should be chosen to maximize the photocurrent generated there. Under the direct spectrum, the model shows $<10 \mathrm{mV}$ change in $V_{\mathrm{oc}}$ of the GaAs junction as the $\mathrm{ZnS}$ thickness is varied: The $\mathrm{ZnS}$ will reduce the reflectance of the luminescence within the $\sim 24^{\circ}$ escape cone, which will tend to reduce the photon recycling enhancement and lower the $V_{\mathrm{oc}}$ of the GaAs junction, but will have no effect on the total internal reflectance of any luminescence outside the escape cone.

For comparison, the upper panel of Fig. 3 also shows the modeled $V_{\text {oc }}$ of the GaAs junction if the upper tandem were still on the substrate (dotted line), with no reflector behind it. For the ideal limiting case of perfect IRE, $V_{\mathrm{oc}}$ increases by $\sim 30 \mathrm{mV}$ due to the photon recycling enhancement effect of the epoxy reflector.

\section{EXPERIMENTAL RESULTS}

Fig. 4 shows the EQE for each junction of a 1-sun fourjunction device. The colors match those in Fig. 2. Bias light was used to separate the responses of the GaInP and GaAs cells, and of the GaInAsP and GaInAs cells, but because the two tandems are not series connected, there is no need to over-bias all three nonlimiting junctions. Strong luminescent coupling can be observed between the two junctions of each tandem, which is evidence of the high IRE [2]. However, because the upper cells were not light-biased when measuring the third junction, no luminescent coupling from the GaAs to the GaInAsP can be discerned in the EQE.

Fig. 5 shows the NREL-certified 1 -sun $I-V$ curves for the fourjunction mechanical stack of Fig. 4, under conditions equiva-

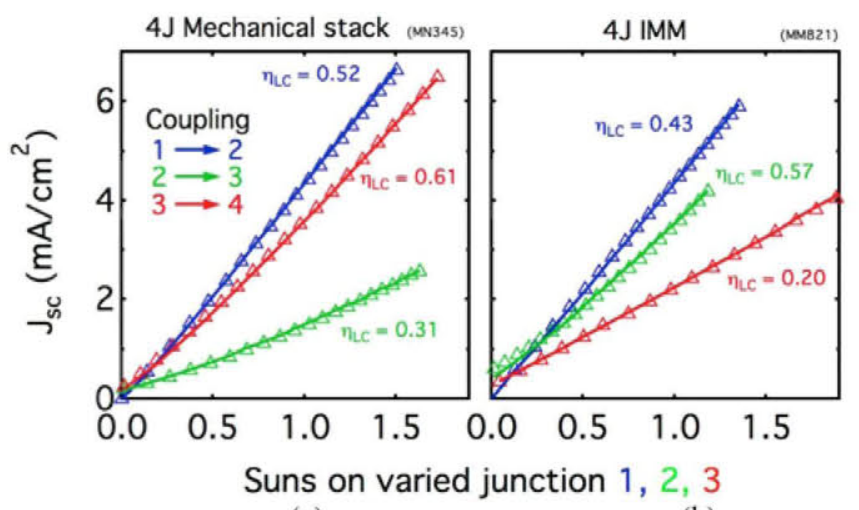

(a)

(b)

Fig. 6. Dark "Suns- $J_{\mathrm{SC}}$ " measurement for four-junction cells: (a) mechanical stack and (b) IMM cell of [14]. The legend indicates which coupling is being measured. In each case, the second junction was not illuminated while the intensity to the first junction was slowly increased; the horizontal axis indicates the relative intensity on the junction being varied. The cells were illuminated with high brightness LEDs. The coupling parameters are shown, as determined by fitting the data to the model in [26].

lent to the G173 global solar spectrum at $1000 \mathrm{~W} / \mathrm{m}^{2}$. Using calibrated reference cells in conjunction with spectral mismatch correction factors, the spectrum was adjusted to give the correct photocurrents in all four junctions, and then the tandems were measured separately. It is important to set the spectrum correctly for all four junctions so that the appropriate amount of luminescent coupling occurs. Despite the enhanced reflectance from the epoxy, there remains some luminescent coupling from the GaAs junction to the GaInAsP junction, and care must be taken to bias the upper tandem correctly when measuring the lower tandem [20]. The correct lower tandem $I-V$ curve is shown in red in Fig. 5, where the upper tandem is biased at $V_{\mathrm{mpp}}$ (or, almost equivalently, at $J_{\mathrm{sc}}$ [20]). If the upper tandem is improperly biased at $V_{\circ C}$ as shown by the green-dashed curve, the lower tandem $J_{\mathrm{sc}}$ increases by $\sim 0.6 \mathrm{~mA} / \mathrm{cm}^{2}$ due to the artificially enhanced luminescent coupling. Under the proper conditions, the cumulative efficiency of the tandem is $30.21 \%+8.54 \%=38.8$ $\pm 1 \%$, which is equal to the best reported efficiencies for twoterminal cells at 1 sun [12]. The uncertainty reflects an estimated $3 \%$ relative uncertainty in the individual measurements.

Fig. 5 also shows the $I-V$ measurement with the second and third contacts shorted to each other so that the device acts as a two-terminal four-junction cell. In that case, the efficiency drops to $\sim 33.9 \%$, largely because the bandgap combination has yielded individual photocurrents that are not all matched. We estimate an additional $\sim 3 \%$ transmission loss due to reflection of incident light at the epoxy interface. With these photocurrents, it is clearly advantageous to operate the cell as a four-terminal device with the two tandems biased at their respective maximum power points.

The two-terminal configuration also allows for a straightforward way to measure the luminescent coupling between adjacent junctions, including the GaAs to GaInAsP coupling. Fig. 6 shows a so-called suns- $J_{\mathrm{sc}}$ measurement, where the irradiance to each junction is systematically varied to force each junction to be limiting, and the overall short-circuit current is recorded. These data were taken on a different but comparable cell to the 
one shown in Fig. 5. Several models of luminescent coupling have been presented in the literature [17], [18], [21]-[26]; the main point here is the relative change in the coupling strength between the various junctions, and we have used the model described in [26] as a guide. The figure shows the effects of coupling between adjacent junctions, where the second junction of each pair was not illuminated while the irradiance to the first junction was slowly increased; the remaining two junctions were heavily light-biased. The data in Fig. 6(a) indicate strong coupling from the first to second junctions (blue curves) and from the third to fourth junctions (red curves), but relatively weaker coupling from the second to third (green curves), as expected, since the intermediate epoxy reflects much of the luminescence and does not allow it to be transmitted. For comparison, we measured a 4J IMM cell in which there is no low-index reflector between the second and third cells. The data in Fig. 6(b) show strong coupling from the first to second junctions, but also strong coupling from the second to third junctions because of the approximate index matching of the semiconductors.

Electroluminescence (EL) was used to measure the ERE for the mechanical stack of Fig. 6(a) and for an IMM with comparable band gaps. In conjunction with optical modeling, the EL measurement enables a determination of the dark currents of each junction [27] and the internal radiative efficiencies [1], [14], as shown in Fig. 7. The emission spectrum from each junction of a solar cell is given approximately by

$$
\Phi_{\mathrm{em}}(\lambda)=\mathrm{EQE}(\lambda) \cdot \phi_{\mathrm{bb}}\left(\lambda ; T_{c}\right)\left[\exp \left(\frac{q V}{k T}\right)-1\right]
$$

where $\phi_{\mathrm{bb}}\left(\lambda ; T_{c}\right)$ is the blackbody distribution within the semiconductor at temperature $T_{c}$, and the ERE is determined from $\int \Phi_{\mathrm{em}}(\lambda) d \lambda / J_{\mathrm{inj}}$ at an injection current $J_{\mathrm{inj}}$. The voltage $V$ in (1) is the diode voltage rather than the total voltage drop, and the expression can, therefore, be inverted to give the diode voltage as a function of the current. We used a spectral evolution highspeed spectrometer to measure the emitted light and calibrated the intensity by measuring the reflection of a known spectrum from a Lambertian surface [5]. The raw data were adjusted so that the calculated optical dark $I-V$ of the series-connected junctions matched the measured electrical dark $I-V$ at low currents where series resistance does not dominate. The data were also adjusted to account for luminescent coupling [25].

As shown in Fig. 7(a) and (b), all four junctions in both the mechanical stack and the IMM are nearly ideal $n=1$ diodes at high currents, and with the exception of the GaAs in the mechanical stack, they are close to ideal even at 1-sun currents. The upper limit of performance can be estimated by the internal radiative limit, shown by the dashed lines in panels (a)-(c). As derived in [1] and [2], the ERE can be expressed by

$$
\eta_{\text {ext }}=\frac{\eta_{\text {int }} \overline{P_{\text {esc }}}}{1-\eta_{\text {int }} \overline{P_{\text {abs }}}}
$$

where $\overline{P_{\text {esc }}}$ is the average probability that an internally emitted photon escapes out the front of the cell, $\overline{P_{\mathrm{abs}}}$ is the average probability that the photon is reabsorbed, and $\eta_{\text {int }}$ is the IRE. A third quantity $\overline{P_{L C}}$ accounts for the probability that the photon is reabsorbed in a lower bandgap junction. $\eta_{\text {ext }} \rightarrow 1$ in the detailed
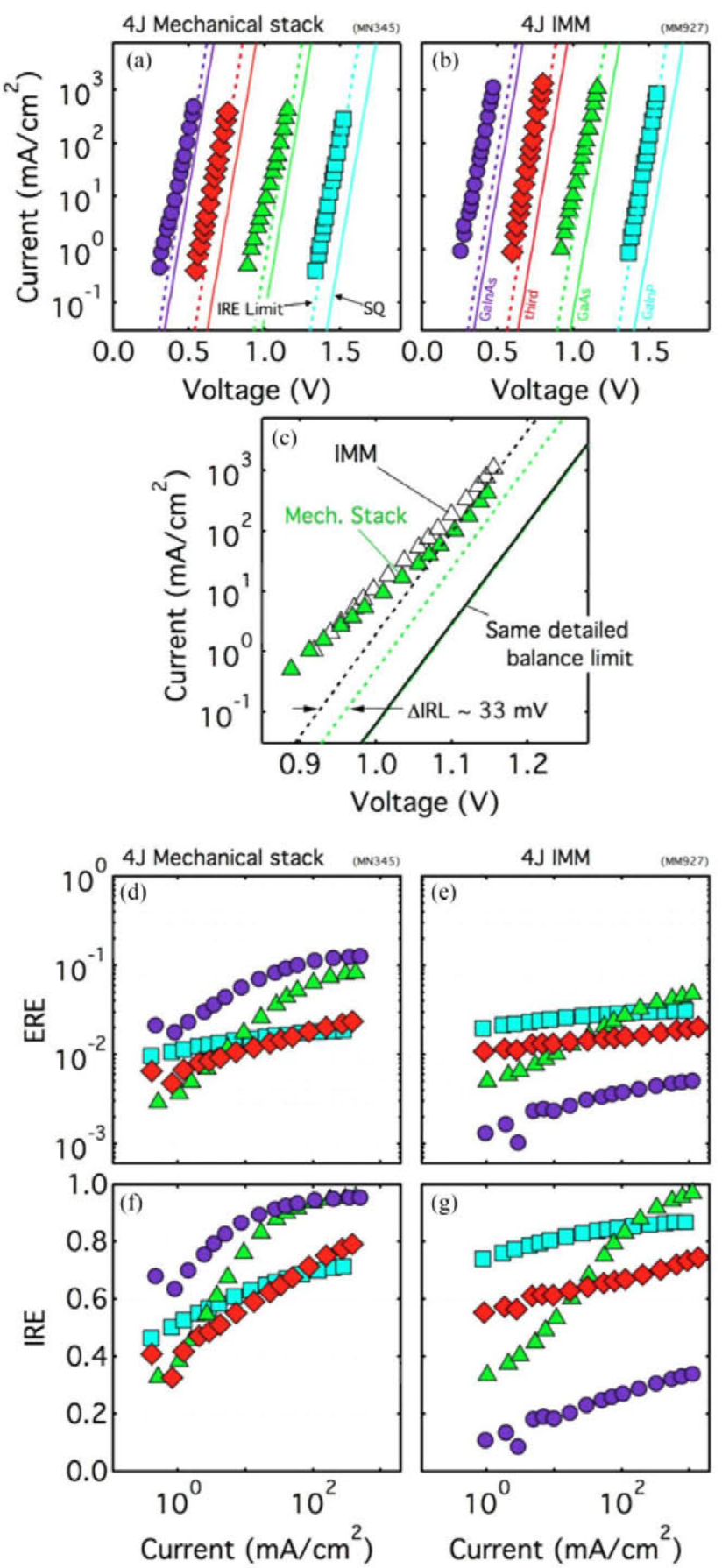

Fig. 7. Recombination currents of the individual junctions are shown as dark $I-V$ curves in panel (a) for a $4 \mathrm{~J}$ mechanical stack and (b) for a $4 \mathrm{~J}$ IMM, with the GaAs junctions magnified in (c). Panels (d) and (e) show the ERE; panels (f) and $(\mathrm{g})$ show the IRE. The junctions are identified in (b) - the third junction is GaInAsP in the mechanical stack, and lattice-mismatched GaInAs in the IMM. In (a)-(c), the solid lines show the detailed balance limit and the dashed lines show the internal radiative limit.

balance limit, but in real cells where $\overline{P_{\mathrm{esc}}}+\overline{P_{\mathrm{abs}}}+\overline{P_{L C}}<1$ because of parasitic optical losses, $\eta_{\text {ext }}<1$, even when $\eta_{\text {int }} \rightarrow 1$. The recombination current when $\eta_{\text {int }} \rightarrow 1$ and $\eta_{\text {ext }}=$ $\frac{\overline{P_{\mathrm{esc}}}}{1-\overline{\mathrm{Pabs}}_{\mathrm{ab}}}$ defines the internal radiative limit. Fig. 7(c) shows a magnified view of the GaAs junctions, and in the mechanical stack, this limit is $\sim 33 \mathrm{mV}$ higher than in the IMM, where there is no 
enhanced photon recycling and is clearly closer to the detailed balance limit shown by the solid lines. Based on optical modeling, we estimate that for the GaAs junction, $\overline{P_{\text {abs }}}$ increases from $\sim 88 \%$ in the IMM to $\sim 97 \%$ in the mechanical stack, while $\overline{P_{\text {esc }}}$ decreases from $0.74 \%$ to $0.61 \%$. The increase in voltage is maintained at lower recombination currents, as would be present at the maximum power point, for example. At the highest currents where the GaAs IREs are comparable, as seen in panels (f) and (g), the GaAs ERE is nearly a factor of 2 higher in panel (d) than in panel (e), again because of the superior internal optics. At 1-sun currents, the actual GaAs voltage in this 4J mechanical stack with a low-index reflector is $\sim 19 \mathrm{mV}$ higher than in the 4J IMM without an intentional reflector.

Other advantages of the mechanical stack are also clear from Fig. 7. The fourth junction in both structures is backed by a gold reflector (behind the semitransparent n-type InP substrate in the case of the mechanical stack), and therefore, the internal optics are good for both cells: the internal radiative limits appear comparable in (a) and (b). However, the IRE in the lattice-matched GaInAs of the mechanical stack is over four times higher, leading to a significantly higher ERE and a voltage increase of $\sim 80 \mathrm{mV}$, from 345 to $425 \mathrm{mV}$. Interestingly, the third junctions are similar in the two structures despite the fact that the GaInAsP is lattice-matched (to InP) in the mechanical stack and the GaInAs is mismatched (with respect to GaAs) in the IMM. Close inspection shows that the IRE and ERE of the GaInAsP are slightly higher at the highest currents, but the voltages are within $10 \mathrm{mV}$ of each other throughout the current range. Rather than implicate the GaInAsP, this testifies to the excellent material quality that has been achieved in the lattice-mismatched GaInAs, demonstrating a threading dislocation density $<10^{6} / \mathrm{cm}^{2}$ [14]. Nevertheless, we expect that the material quality in the quaternary could still be improved, driving a higher voltage in the mechanical stack.

Fig. 8 shows the $I-V$ curves and efficiency of a similar concentrator cell under flash illumination, with the upper tandem biased at $J_{\mathrm{sc}}$ during the measurement of the lower tandem; as shown in [20], there is little difference between biasing the upper tandem at $J_{\mathrm{sc}}$ or $V_{\mathrm{mpp}}$, for well-behaved cells. $I-V$ curves were also measured with the upper tandem biased at $V_{\text {oc }}$ to show the effects of luminescent coupling. The data were acquired on a High-Intensity Pulsed Solar Simulator (HIPSS); the upper junctions were reasonably matched to the reference spectrum, but the lower junctions were mismatched due to the inability to finetune the spectrum, and the lower tandem efficiency is therefore more uncertain. The concentration was determined by assuming linearity of the photocurrent with the incident intensity. This cell had a cumulative 1-sun efficiency under the direct spectrum of $28.0 \%+6.87 \%=34.9 \%$. For the upper tandem, the efficiency rolls over at relatively low concentration, likely due to an unacceptably high series resistance. The lower tandem efficiency peaks at $\sim 240$ suns, and the cumulative efficiency reaches a peak of $42 \pm 2 \%$ at $\sim 100$ suns, although this efficiency is uncorrected for spectral error [28] and is most likely an overestimate.

The upper panel of Fig. 8 shows a substantial difference in the bottom tandem photocurrent between the cases where the upper tandem is biased at $V_{\mathrm{oc}}$ (green dashes) and $J_{\mathrm{sc}}$ (red lines),
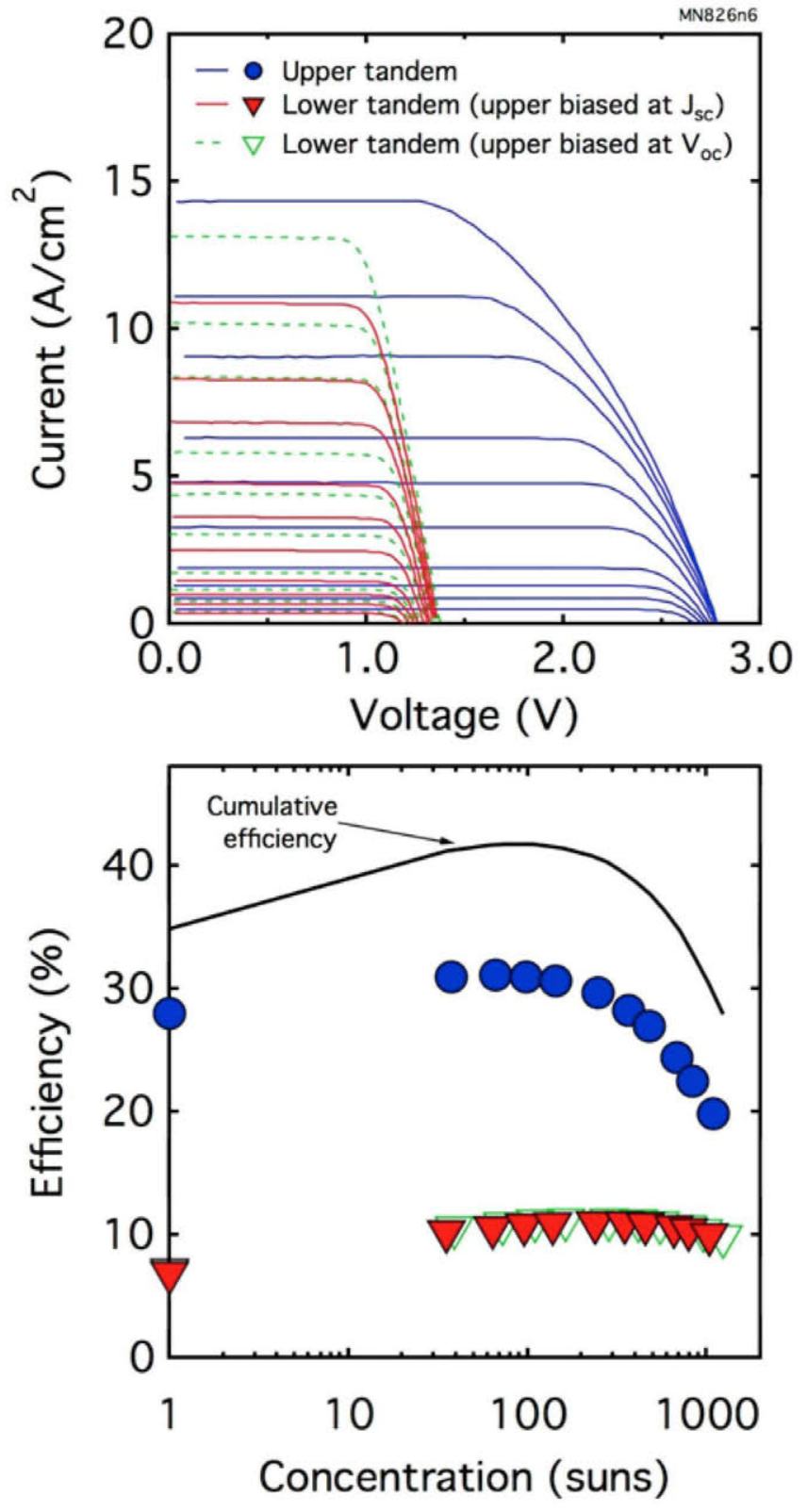

Fig. 8. Concentrator $I-V$ curves and efficiency, measured on the HIPSS. The upper panel shows the $I-V$ curves, with the intensity increasing from bottom to top. The lower panel shows the extracted efficiencies as a function of the quantified intensity. The color scheme is the same in both panels. The lower tandem was measured with the upper tandem at both open-circuit (green) and shortcircuit (red), as indicated; the cumulative efficiency is based on the latter. Based on the nominal flash spectrum, the photocurrent ratios were 1:0.93:1.3:0.72 relative to the G173 direct reference spectrum. The 1-sun data were taken on a Class A adjustable solar simulator.

indicating that the luminescent coupling persists to high concentrations, as expected.

\section{DISCUSSION}

The data in Figs. 4-8 show that four-terminal mechanically stacked solar cells can be fabricated with very high efficiencies. The intermediate optical layers can be tuned to boost the photon recycling in the upper junctions, while maintaining a high transmission of incident light to the lower junctions. Fig. 7 
clearly shows a higher internal radiative limit for the second junction of the mechanical stack compared with the second junction of the IMM. Although the upper tandem in Fig. 5 showed only a modest boost in $V_{\mathrm{oc}}$, the potential for an enhancement is clear.

For comparison, Sheng et al. [10] reported a $\mathrm{GaInP} / \mathrm{GaAs} / \mathrm{GaInAsNSb} / \mathrm{Ge}$ mechanical stack that was fabricated by a transfer printing technique, with a cumulative four-terminal 1-sun efficiency of $32.9 \%$ and a peak concentrator efficiency of $43.9 \%$ at 1000 suns. The printing technique relies in part on an index-matched $\mathrm{As}_{2} \mathrm{Se}_{3}$ layer to maximize the optical transmission between the components, without enabling any enhanced photon recycling in the upper cells. Dimroth et al. [11] reported a series-connected 4J wafer-bonded cell with similar bandgaps to those reported here and demonstrated a 1 -sun efficiency of $34.5 \pm 1.7 \%$. This efficiency is slightly higher but comparable with the two-terminal efficiency of $33.9 \%$ in Fig. 5, but those authors note that the bandgaps were adjusted to better match the photocurrents. Because the mechanical stack involves a direct semiconductor bond, there is again no opportunity to enhance the photon recycling in the upper junctions. A similar wafer-bonded architecture was reported for a five-junction cell by Chiu et al. [13], demonstrating a two-terminal 1-sun efficiency of $37.8 \%$.

The concentrator cells developed here have excellent 1-sun performance. The efficiency under concentrated light is $>40 \%$, but rolls over at a relatively low concentration due to an unknown series resistance which is being investigated. With improvements, we expect efficiencies $>45 \%$ to be attainable. A gridded external contact will present series resistance design challenges in any solar cell, but in the four-terminal configuration, there are three gridded contacts rather than one. We have done extensive tests and measurements to assess the contributions to the series resistance. Different metallization patterns were designed for target concentrations of either 300 or 500 suns, based on the measured sheet resistances of $120 \Omega / \mathrm{sqr}$ in the GaInP emitter, $30 \Omega /$ sqr in the AlGaAs LCL behind the GaAs junction, and $5 \Omega / \mathrm{sqr}$ in the LCL in front of the GaInAsP junction. The specific contact resistance between the metal and semiconductor was found to be $<10^{-4} \Omega \cdot \mathrm{cm}^{2}$ on test structures for all four contacts, but determining the contact resistance in the actual device can be difficult. Based on repeated difficulties electroplating metal to the front contact layer, we believe that an unacceptably high contact resistance at the front surface is the dominant cause for efficiency rollover in the present cells.

The high efficiency achieved at 1-sun, especially for the upper GaInP/GaAs tandem, suggests a relevance to a promising 1sun and low-concentration operational space. For any III-V cell to be cost effective at 1-sun, the substrate will have to be removed and reused (many times), with the epilayers bonded to a secondary handle. Rather than just a passive supporting layer, that handle could be an active solar cell fabricated from a lower bandgap semiconductor, thereby forming a mechanical stack. Our results here indicate that a low-index transparent adhesive would boost the performance of that 1-sun device, and the additional contribution of the power generation from the active handle would further increase the efficiency. We recently demonstrated preliminary GaInP//silicon tandems based on the fabrication methods discussed here [15]. The ideal active handle would have a direct and tunable bandgap, be inexpensive to deposit and process, and be able to be deposited on a flexible surface so that it could be part of the III-V substrate removal process. Tradeoffs among these ideal characteristics may be necessary, but in this way, the technology demonstrated here may find broader application beyond high concentrator solar cells.

In summary, we have demonstrated mechanically stacked four-junction solar cells based on junctions grown on GaAs and InP substrates. A low-index epoxy was used to bond the components together and reflect a substantial portion of the internal luminescence in the GaAs cell. As deduced by EL, the internal radiative limit of the GaAs junction increases by $>30 \mathrm{mV}$ because of the additional reflectance, and the actual junction voltage increased by $\sim 20 \mathrm{mV}$. Efficiencies of $38.8 \pm 1.0 \%$ under 1 -sun global conditions and $\sim 42 \%$ under 100 -sun direct concentrator conditions were achieved. Even higher concentrator efficiencies are expected when series resistance limitations are overcome

\section{ACKNOWLEDGMENT}

The authors are pleased to thank Prof. E. Yablonovitch for useful and inspiring conversations, T. Moriarty for certified $I-V$ measurements, and V. LaSalvia for processing assistance.

\section{REFERENCES}

[1] M. A. Steiner et al., "Optical enhancement of the open-circuit voltage in high quality GaAs solar cells," J. Appl. Phys., vol. 113, art. no. 123109, 2013

[2] M. A. Steiner et al., "Effects of internal luminescence and internal optics on Voc and Jsc of III-V solar cells," IEEE J. Photovoltaics, vol. 3, no. 4, pp. 1437-1442, Oct. 2013.

[3] O. D. Miller, E. Yablonovitch, and S. R. Kurtz, "Strong internal and external luminescence as solar cells approach the Shockley-Queisser limit," IEEE J. Photovoltaics, vol. 2, no. 3, pp. 303-311, Jul. 2012.

[4] B. M. Kayes et al., " $27.6 \%$ conversion efficiency, a new record for singlejunction solar cells under 1 sun illumination," in Proc. 37th IEEE Photovoltaic Spec. Conf., Seattle, WA, USA, 2011, pp. 4-8.

[5] J. F. Geisz, M. A. Steiner, I. Garcia, S. R. Kurtz, and D. J. Friedman, "Enhanced external radiative efficiency for $20.8 \%$ efficient single-junction GaInP solar cells," Appl. Phys. Lett., vol. 103, art. no. 041118, 2013.

[6] V. Ganapati, C.-S. Ho, and E. Yablonovitch, "Intermediate mirrors to reach theoretical efficiency limits of multi-bandgap solar cells," IEEE $J$. Photovoltaics, vol. 5, no. 1, pp. 410-417, Jan. 2015.

[7] A. Marti and G. L. Araujo, "Limiting efficiencies for photovoltaic energy conversion in multigap systems," Sol. Energy Mater. Sol. Cells, vol. 43, pp. 203-222, 1996.

[8] I. Garcia et al., "Design of semiconductor-based back reflectors for high Voc monolithic multijunction solar cells," presented at the 38th IEEE Photovoltaic Spec. Conf., Austin, TX, USA, 2012.

[9] I. Garcia et al., "Back reflectors based on buried $\mathrm{Al}_{2} \mathrm{O}_{3}$ for enhancement of photon recycling in monolithic, on-substrate III-V solar cells," Appl. Phys. Lett., vol. 105, art. no. 133507, 2014.

[10] X. Sheng et al., "Printing-based assembly of quadruple-junction fourterminal microscale solar cells and their use in high-efficiency modules," Nature Mater, vol. 13, pp. 593-598, 2014

[11] F. Dimroth et al., "Wafer bonded four-junction GaInP/GaAs//GaInAsP/ GaInAs concentrator solar cells with 44.7\% efficiency," Prog. Photovoltaics, vol. 22 , pp. 277-282, 2014.

[12] P. T. Chiu et al., "Direct semiconductor bonded 5J cell for space and terrestrial applications," IEEE J. Photovoltaics, vol. 4, no. 1, pp. 493-497, Jan. 2014. 
[13] P. T. Chiu et al., "35.8\% space and $38.8 \%$ terrestrial $5 \mathrm{~J}$ direct bonded cells," in Proc. 40th IEEE Photovoltaic Spec. Conf., Denver, CO, USA, 2014, pp. 0011-0013.

[14] R. M. France et al., "Quadruple junction inverted metamorphic concentrator devices," IEEE J. Photovoltaics, vol. 5, no. 1, pp. 432-437, Jan. 2015.

[15] S. Essig et al., "Progress towards a 30\% efficiency GaInP/Si tandem solar cell," Energy Procedia, vol. 77, pp. 464-469, 2015.

[16] A. S. Brown and M. A. Green, "Radiative coupling as a means to reduce spectral mismatch in monolithic tandem solar cell stacks-theoretical considerations," in Proc. 29th IEEE Photovoltaic Spec. Conf., New Orleans, LA, USA, 2002, pp. 868-871.

[17] M. A. Steiner and J. F. Geisz, "Non-linear luminescent coupling in seriesconnected multijunction solar cells," Appl. Phys. Lett., vol. 100, art. no. $251106,2012$.

[18] C. Baur, M. Hermie, F. Dimroth, and A. W. Bett, "Effects of optical coupling in III-V multilayer systems," Appl. Phys. Lett., vol. 90, art. no. $192109,2007$.

[19] J. F. Geisz et al., "High-efficiency GaInP/GaAs/InGaAs triple-junction solar cells grown inverted with a metamorphic bottom junction," Appl. Phys. Lett., vol. 91, art. no. 023502, 2007.

[20] M. A. Steiner et al., "A monolithic three-terminal GaInAsP/GaInAs Tandem solar cell," Prog. Photovoltaics, vol. 17, pp. 587-593, 2009.

[21] H. Yoon, R. R. King, G. Kinsey, S. Kurtz, and D. D. Krut, "Radiative coupling effects in GaInP/GaAs/Ge multijunction solar cells," in Proc. 3rd World Conf. Photovoltaic Energy Convers., Osaka, Japan, 2003, pp. $745-748$.
[22] S. H. Lim, J.-J. Li, E. H. Steenbergen, and Y.-H. Zhang, "Luminescence coupling effects on multijunction solar cell external quantum efficiency measurement," Prog. Photovoltaics, vol. 21, pp. 344-350, 2011.

[23] T. Sogabe et al., "Experimental characterization and self-consistent modeling of luminescence coupling effects in III-V multijunction solar cells," Appl. Phys. Lett., vol. 103, art. no. 263907, 2013.

[24] D. Derkacs, D. R. Bilir, and V. A. Sabnis, "Luminscent coupling in GaAs/GaInNAsSb multijunction solar cells," IEEE J. Photovoltaics, vol. 3, no. 1, pp. 520-527, Jan. 2013.

[25] J. F. Geisz et al., "Generalized optoelectronic model of series-connected multijunction solar cells," IEEE J. Photovoltaics, vol. 5, no. 6, pp. 1827-1839, Nov. 2015.

[26] M. A. Steiner et al., "Measuring IV curves and Subcell photocurrents in the presence of luminescent coupling," IEEE J. Photovoltaics, vol. 3 , no. 2, pp. 879-887, Apr. 2013.

[27] S. Roensch, R. Hoheisel, F. Dimroth, and A. W. Bett, "Subcell I-V characteristic analysis of $\mathrm{GaInP} / \mathrm{GaInAs} / \mathrm{Ge}$ solar cells using electroluminescence measurements," Appl. Phys. Lett., vol. 98, art. no. 251113, 2011.

[28] C. R. Osterwald, M. W. Wanlass, T. Moriarty, M. A. Steiner and K. A. Emery, "Empirical procedure to correct concentrator cell efficiency measurement errors caused by unfiltered Xenon flash solar simulators," presented at the 40th IEEE Photovoltaic Spec. Conf., Denver, CO, USA, 2014. 